PROCEEDINGS OF THE

AMERICAN MATHEMATICAL SOCIETY

Volume 124, Number 7, July 1996

\title{
MULTINOMIAL EXPANSIONS AND THE PYTHAGOREAN THEOREM
}

\author{
ALLAN FRYANT
}

(Communicated by J. Marshall Ash)

Dedicated to the memory of Professor Morris Marden

\begin{abstract}
An addition formula for homogeneous polynomials is used to obtain a generalization of the Pythagorean theorem and a new view of the multinomial expansion.
\end{abstract}

Let $x=\left(x_{1}, x_{2}, \ldots, x_{n}\right) \in \mathbf{R}^{n}$, and let $P$ denote the set of all polynomials

$$
p(x)=\sum_{j=1}^{m} a_{j} x_{1}^{\alpha_{j}^{1}} x_{2}^{\alpha_{j}^{2}} \ldots x_{n}^{\alpha_{j}^{n}}, \quad a_{j} \in \mathbf{R} .
$$

Letting $\partial / \partial x=\left(\partial / \partial x_{1}, \partial / \partial x_{2}, \ldots, \partial / \partial x_{n}\right)$, we define on $P$ the inner product

$$
\begin{aligned}
(p, q) & =\left.p\left(\frac{\partial}{\partial x}\right) q(x)\right|_{x=0} \\
& =\left.\left(\sum_{j=1}^{m} a_{j} \frac{\partial^{\alpha_{j}^{1}}}{\partial x_{1}^{\alpha_{j}^{1}}} \frac{\partial^{\alpha_{j}^{2}}}{\partial x_{2}^{\alpha_{j}^{2}}} \ldots \frac{\partial^{\alpha_{j}^{n}}}{\partial x_{n}^{\alpha_{j}^{n}}}\right) q(x)\right|_{x=0} .
\end{aligned}
$$

Let $H_{k} \subset P$ denote the vector space of homogeneous polynomials of degree $k$. Note that the dimension of $H_{k}$ is

$$
\operatorname{dim} H_{k}=d_{k}=\frac{(n+k-1) !}{(n-1) ! k !} .
$$

We first obtain a simple analog of the Funk-Hecke theorem [1, p. 247] for homogeneous polynomials.

Theorem 1. If $p(x)$ is a homogeneous polynomial of degree $k$, then

$$
\left(\left(x_{1} y_{1}+x_{2} y_{2}+\cdots+x_{n} y_{n}\right)^{k}, p(y)\right)=k ! p(x) .
$$

Proof. Expanding the power,

$$
\begin{aligned}
\left(x_{1} y_{1}+\cdots+x_{n} y_{n}\right)^{k} & =\sum_{\alpha} \frac{k !}{\alpha_{1} ! \alpha_{2} ! \ldots \alpha_{n} !}\left(x_{1} y_{1}\right)^{\alpha_{1}}\left(x_{2} y_{2}\right)^{\alpha_{2}} \ldots\left(x_{n} y_{n}\right)^{\alpha_{n}} \\
& =k ! \sum_{\alpha} \frac{x_{1}^{\alpha_{1}} x_{2}^{\alpha_{2}} \ldots x_{n}^{\alpha_{n}}}{\alpha_{1} ! \alpha_{2} ! \ldots \alpha_{n} !} y_{1}^{\alpha_{1}} y_{2}^{\alpha_{2}} \ldots y_{n}^{\alpha_{n}} .
\end{aligned}
$$

Received by the editors October 7, 1994.

1991 Mathematics Subject Classification. Primary 33E99; Secondary 26 C99.

Key words and phrases. Homogeneous polynomials, addition formula. 
Thus if $p(y)=\sum_{\alpha} a_{\alpha} y_{1}^{\alpha_{1}} y_{2}^{\alpha_{2}} \ldots y_{n}^{\alpha_{n}}$, we have

$$
\begin{aligned}
& \left(\left(x_{1} y_{1}+\cdots+x_{n} y_{n}\right)^{k}, p(y)\right) \\
& =\left(k ! \sum_{\alpha} \frac{x_{1}^{\alpha_{1}} \cdots x_{n}^{\alpha_{n}}}{\alpha_{1} ! \ldots \alpha_{n} !} \frac{\partial^{\alpha_{1}}}{\partial y_{1}^{\alpha_{1}}} \ldots \frac{\partial^{\alpha_{n}}}{\partial y_{n}^{\alpha_{n}}} \sum_{\beta} a_{\beta} y_{1}^{\beta_{1}} \ldots y_{n}^{\beta_{n}}\right) \\
& =k ! \sum_{\alpha} a_{\alpha} x_{1}^{\alpha_{1}} \ldots x_{n}^{\alpha_{n}}=k ! p(x) .
\end{aligned}
$$

Our next result is an addition formula for orthonormal homogeneous polynomials.

Theorem 2 (Addition Formula). Let $\left\{p_{j}(x)\right\}_{j=1}^{d_{k}}$ be an orthonormal basis for $H_{k}$. Then

$$
\left(x_{1} y_{1}+x_{2} y_{2}+\cdots+x_{n} y_{n}\right)^{k}=k ! \sum_{j=1}^{d_{k}} p_{j}(x) p_{j}(y) .
$$

Proof. Let $x=\left(x_{1}, x_{2}, \ldots, x_{n}\right)$ and $\alpha_{j}=\left(\alpha_{j}^{1}, \alpha_{j}^{2}, \ldots, \alpha_{j}^{n}\right)$. For convenience we write $x^{\alpha_{j}}=x_{1}^{\alpha_{j}^{1}} x_{2}^{\alpha_{j}^{2}} \ldots x_{n}^{\alpha_{j}^{n}}$. Then expanding the power, we have

$$
\left(x_{1} y_{1}+\cdots+x_{n} y_{n}\right)^{h}=\sum c_{j} x^{\alpha_{j}} y^{\alpha_{j}},
$$

where $x^{\alpha_{j}}$ and $y^{\alpha_{j}}$ are homogeneous monomials of degree $k$. Since $\left\{p_{j}(x)\right\}_{j=1}^{d_{k}}$ is a basis for $H_{k}$, there exist constants $a_{j m}$ such that

$$
y^{\alpha_{j}}=\sum_{m=1}^{d_{k}} a_{j m} p_{m}(y) \text {. }
$$

Thus

$$
\begin{aligned}
\left(x_{1} y_{1}+\cdots+x_{n} y_{n}\right)^{k} & =\sum_{j} c_{j} x^{\alpha_{j}}\left(\sum_{m} a_{j m} p_{m}(y)\right) \\
& =\sum_{j} g_{j}(x) p_{j}(y),
\end{aligned}
$$

where the last expression is a rearrangement of the previous sum. The $g_{j}(x)$ are linear combinations of the monomials $x^{\alpha_{j}}$, and thus are homogeneous polynomials of degree $k$ in $x$. Further, since the $p_{j}(x)$ are orthonormal,

$$
\left(\left(x_{1} y_{1}+\cdots+x_{n} y_{n}\right)^{k}, p_{l}(y)\right)=\left(\sum_{j} g_{j}(x) p_{j}(y), p_{l}(y)\right)=g_{l}(x) .
$$

But by the identity given in Theorem 1 ,

$$
\left(\left(x_{1} y_{1}+\cdots+x_{n} y_{n}\right)^{k}, p_{l}(y)\right)=k ! p_{l}(x) .
$$

Thus,

$$
g_{j}(x)=k ! p_{j}(x), \quad j=1,2, \ldots, d_{k},
$$

which completes the proof. 
If $\alpha_{j}=\left(\alpha_{j}^{1}, \alpha_{j}^{2}, \ldots, \alpha_{j}^{n}\right)$, for convenience we write $\alpha_{j} !=\alpha_{j}^{1} ! \alpha_{j}^{2} ! \ldots \alpha_{j}^{n} !$. The result of Theorem 2 shows that the multinomial expansion

$$
\left(x_{1} y_{1}+x_{2} y_{2}+\cdots+x_{n} y_{n}\right)^{k}=\sum \frac{k !}{\alpha_{j} !} x^{\alpha_{j}} y^{\alpha_{j}}=k ! \sum \frac{x^{\alpha_{j}}}{\sqrt{\alpha_{j} !}} \frac{y^{\alpha_{j}}}{\sqrt{\alpha_{j} !}}
$$

is no more than an addition formula for the orthonormal basis $\left\{x^{\alpha_{j}} / \sqrt{\alpha_{j} !}\right\}_{j=1}^{d_{k}}$ of $H_{k}$. The significance of the result (2) lies with the fact that it holds for any orthonormal basis $\left\{p_{j}(x)\right\}_{1}^{d_{k}}$, not merely the monomials $\left\{x^{\alpha_{j}} / \sqrt{\alpha_{j}}\right\}_{j=1}^{d_{k}}$.

The addition formula of Theorem 2 yields an identity which contains the Pythagorean theorem $\sin ^{2} \theta+\cos ^{2} \theta=1$ as a special case:

Theorem 3 (Pythagorean Identity). Suppose $\left\{p_{j}(x)\right\}_{j=1}^{d_{k}}$ is an orthonormal basis for $H_{k}$. Then on the unit sphere $|s|=\sqrt{s_{1}^{2}+s_{2}^{2}+\cdots+s_{n}^{2}}=1$,

$$
\sum_{j=1}^{d_{k}}\left[p_{j}(s)\right]^{2}=\frac{1}{k !} .
$$

Proof. Letting $x=y$ in the addition formula yields

$$
\left(x_{1}^{2}+x_{2}^{2}+\cdots+x_{n}^{2}\right)^{k}=k ! \sum_{j=1}^{d_{k}}\left[p_{j}(x)\right]^{2},
$$

from which the result is immediate.

Most inner products are integrals. Although the inner product we have used here is defined by a derivative, it is far from artificial. Indeed, this inner product allows us to view Taylor series as Fourier series. That is, suppose we have the Taylor series

$$
f(x)=\sum_{j=0}^{\infty} a_{j} x^{\alpha_{j}} .
$$

Rewriting the series as

$$
f(x)=\sum_{j=1}^{\infty} b_{j} \frac{x^{\alpha_{j}}}{\sqrt{\alpha_{j} !}},
$$

and noting that the monomials $\left\{x^{\alpha_{j}} / \sqrt{\alpha_{j} !}\right\}_{j=1}^{\infty}$ are orthonormal with respect to the inner product (1), we have

$$
\left(x^{\alpha_{j}} / \sqrt{\alpha_{j} !}, f(x)\right)=b_{j} .
$$

These simple results appear to have considerable analytic significance. To see this, one need only consider the kernel

$$
\sum_{k=0}^{\infty} \sum_{j=1}^{d_{k}} p_{k}^{j}(x) p_{k}^{j}(y)=\sum_{k=0}^{\infty} \frac{(x \cdot y)^{k}}{k !}=e^{x \cdot y},
$$

and the reproducing formula

$$
\left(f(y), e^{x \cdot y}\right)=f(x) .
$$

Here $\left\{p_{k}^{j}(x)\right\}_{j=1}^{d_{k}}, k=0,1,2, \ldots$, are arbitrary orthonormal bases for the homogeneous polynomials $H_{k}$ of degree $k$. 


\section{REFERENCES}

1. A. Erdelyi, Higher transcendental functions, Vol. 2, McGraw-Hill, New York, 1953. MR 15:419i Department of Mathematics, Greensboro College, Greensboro, North Carolina 27401 